\title{
Verification of Architectural Refactorings by Rule Extraction
}

\author{
Dénes Bisztray ${ }^{1}$, Reiko Heckel ${ }^{1}$, and Hartmut Ehrig ${ }^{2}$ \\ ${ }^{1}$ Department of Computer Science, University of Leicester \\ $\{$ dab24, reiko\}@mcs.le.ac.uk \\ ${ }^{2}$ Institut für Softwaretechnik und Theoretische Informatik, \\ Technische Universität Berlin \\ ehrig@cs.tu-berlin.de
}

\begin{abstract}
With the success of model-driven development as well as component-based and service-oriented systems, models of software architecture are key artefacts in the development process. To adapt to changing requirements and improve internal software quality such models have to evolve while preserving aspects of their behaviour.

To avoid the costly verification of refactoring steps on large systems we present a method which allows us to extract a (usually much smaller) rule from the transformation performed and verify this rule instead. The main result of the paper shows that the verification of rules is indeed sufficient to guarantee the desired semantic relation between source and target models. We apply the approach to the refactoring of architectural models based on UML component, structure, and activity diagrams, with using CSP as a semantic domain.
\end{abstract}

Keywords: Service Oriented Architecture, UML, Refactoring, Graph Transformation, CSP.

\section{Introduction}

Nothing endures but change, as the philosopher says Lae25. As much as anywhere else, this applies to the world of software. In order to improve the internal structure, performance, or scalability of software systems, changes may be required that preserve the observable behaviour of systems. In OO programming, such behaviour-preserving transformations are known as refactorings $\left[\mathrm{FBB}^{+} 99\right.$. Today, where applications tend to be distributed and service-oriented, the most interesting changes take place at the architectural level. Even if these changes are structural, they have to take into account the behaviour encapsulated inside the components that are being replaced or reconnected. In analogy to the programming level we speak of architectural refactorings if preservation of observable behaviour is intended.

In this paper, refactoring is addressed at the level of models. Given a transformation from a source to a target model we would like to be able to verify their relation. In order to make this precise we have to fix three ingredients: the modelling language used, its semantics, and the relation capturing our idea of 
behaviour preservation. Notice however that in the mathematical formulation of our approach, these parameters can be replaced by others, subject to certain requirements. For modelling language we use the UML, which provides the means to describe both structure (by component and static structure diagrams) and behaviour (by activity diagrams) of service-oriented systems OMG06. The semantics of the relevant fragment of the UML is expressed in a denotational style, using CSP Hoa85 as semantic domain and defining the mapping from UML diagrams to CSP processes by means of graph transformation rules. As different UML diagrams are semantically overlapping, the mapping has to produce one single consistent semantic model EKGH01. The semantic relation of behaviour preservation can conveniently be expressed using one of the refinement and equivalence relations on CSP processes.

Based on these (or analogue) ingredients, we can formalise the question by saying that a model transformation $M_{1} \rightarrow M_{2}$ is behaviour-preserving if $\operatorname{sem}\left(M_{1}\right)$ $\mathcal{R} \operatorname{sem}\left(M_{2}\right)$ where sem represents the semantic mapping and $\mathcal{R}$ the desired relation on the semantic domain. However, the verification of relation $\mathcal{R}$ over sufficiently large $M_{1}$ and $M_{2}$ can be very costly, while the actual refactoring might only affect a relatively small fragment of the overall model. Hence, it would be advantageous if we could focus our verification on those parts of the model that have been changed, that is, verify the refactoring rules rather than the actual steps. This is indeed possible, as we show in this paper, if both semantic mapping sem and semantic relation $\mathcal{R}$ satisfy suitable compositionality properties. We satisfy these requirements by specifying the mapping sem by graph transformation rules of a certain format and choosing CSP refinements as semantic relations.

However, model-level architectural refactorings are unlikely to be created directly from semantics-preserving rules. Such rule catalogues as exist focus on object-oriented systems and are effectively liftings to the model level of refactoring rules for OO programs. Rather, an engineer using a modelling tool performs a manual model transformation $M_{1} \rightarrow M_{2}$ from which a verifiable refactoring rule has to be extracted first. In this we follow the idea of model transformation by example Var06 where model transformation rules expressed as graph transformations are derived from sample transformations.

The paper is structured as follows. In Sect. 3 we present our architectural models along with an example, on which a refactoring step is performed in Sect.4 Section 5 introduces CSP as the semantic domain and describes the mapping and the semantic relation. The formal justification for rule-level verification is discussed in Sect. 6. It is demonstrated that the method is sound if the semantic mapping is compositional, which is true based on a general result which derives this property from the format of the mapping rules. Section 7 concludes the paper. A detailed exposition of all relevant definitions and proofs is given in Bis08.

\section{Related Work}

After refactorings for Java were made popular by Fowler $\mathrm{FBB}^{+} 99$, several proposals for formalisation and verification based on first-order logics and invariants 
have been made SPTJ01LM04MGB06. The first formal approach to refactoring based on graph transformations is due to Mens [MDJ02, focussing on the analysis of conflicts and dependencies between rules.

Refactoring of architectural models has been studied formally in architectural description languages (ADLs) like Wright ADG98 or Darwin [MK96, using process calculi like CSP or $\pi$-calculus for expressing formal semantics. Our semantic mapping to CSP follows that of [EKGH01 for UML-RT Sel98, an earlier component-based extension to the UML, but distinguishes type and instance level architectural models in UML 2.

A number of authors have studied instance level architectural transformations, or reconfigurations. For example, Taentzer TGM00 introduces the notion of distributed graph transformation systems to allow architectural reconfiguration by means of two-level rules to express system-level and local transformations. The approach of WF02 uses an algebraic framework to represent reconfigurations based on the coordination language Community. In HIM98 the architecture is represented by hypergraphs, where the hyperedges are the components, and the nodes are the communication ports. Architectural reconfigurations are represented by synchronised hyperedge replacement rules.

Our approach combines the type level, typical of source code refactoring, which happens at the level of classes, with the instance level typical of architectural transformations.

\section{Architectural Models}

This section presents our choice of architectural modelling language by means of an example based on the Car Accident Scenario from the SENSORIA Automotive Case Study $\mathrm{WCG}^{+} 06$.

We use UML component and composite structure diagrams for representing the type and instance-level architecture of our system in conjunction with activity diagrams specifying the workflows executed by component instances OMG06.

Briefly, the scenario is as follows. A car company is offering a service by which, in case one of the sensors in their car detects an accident, customers are contacted via their mobile phone to check if they require assistance. If they do, a nearby ambulance is dispatched. The system consists of three main parts: the agent in the car, the accident server, and the interface to the local emergency services. We present the architecture and behaviour of the accident server in detail.

Type-Level. Component diagrams specify the components, ports, interfaces that make up the building blocks of the system. Figure 1(a) shows the component diagram of the accident server.

The AccidentManager is the core component, responsible for receiving incoming alerts from cars through the AlertRecv port. In order to initiate a phone call it acquires the number of the driver from the PersistentDatabase, and passes it to the PhoneService, which calls the driver. In case the driver replies saying that assistance is not required, the alert is cancelled. Otherwise, the call is returned to 


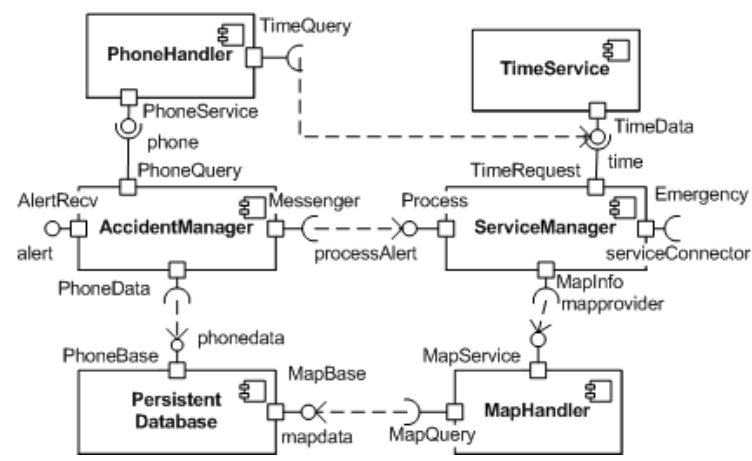

(a) Component Diagram

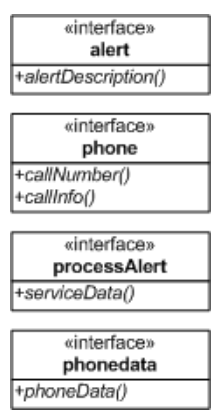

(b) Interfaces

Fig. 1. Architectural model of the accident server

the AccidentManager, which assesses the available data (including sensorial and location data from the car) and decides if the situation is a real emergency. In this case it passes the necessary data to the ServiceManager, which matches the GPS location of the car using the MapHandler, creates a service description, and contacts the serviceConnector interface that provides access to local emergency services.

In the diagram, components are represented by rectangles with a component icon and classifier name. Smaller squares on the components represent the ports, provided interfaces are represented by circles and required interfaces by a socket shape OMG06. Dashed arrows represent dependencies between the provided and required interfaces.

Instance-Level. The composite structure diagram specifying the configuration of the accident server is shown in Figure 2. Boxes named instance : type represent component instances. Ports are typed by interfaces defining the possible actions that can happen through that port. For instance, the possible actions of the PhoneQuery port are defined by the phone interface. Links between port instances represent connectors, enabling communication between component instances OMG06.

Behaviour. The behaviours of components are described by activity diagrams, like the one depicted in Figure 3 associated with the AccidentManager component. Apart from the obvious control flow constructs they feature accept event actions, denoted by concave pentagons, that wait for the occurrence of specific events triggered by send signal actions, shown as convex pentagons OMG06. They fit into the communication framework by representing functions calls from the corresponding interface through the relevant port. For instance, the phoneData send signal action in Fig. 3represents the function call from phone interface through PhoneQuery port. 


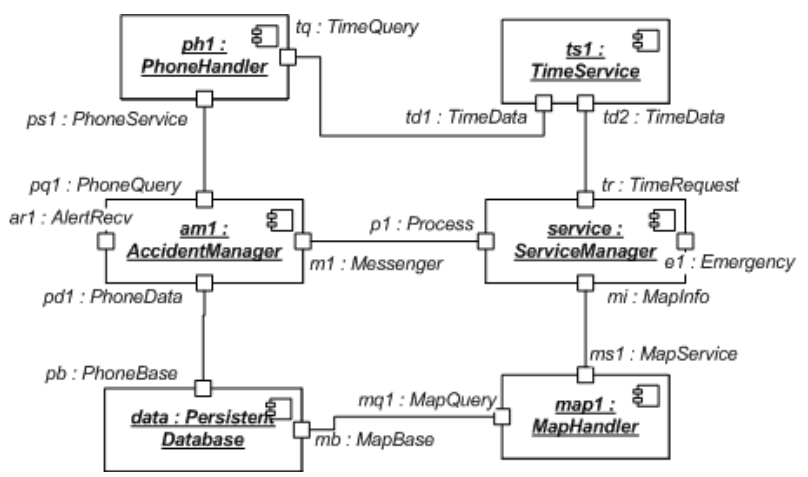

Fig. 2. Static Structure Diagram of the Accident Server

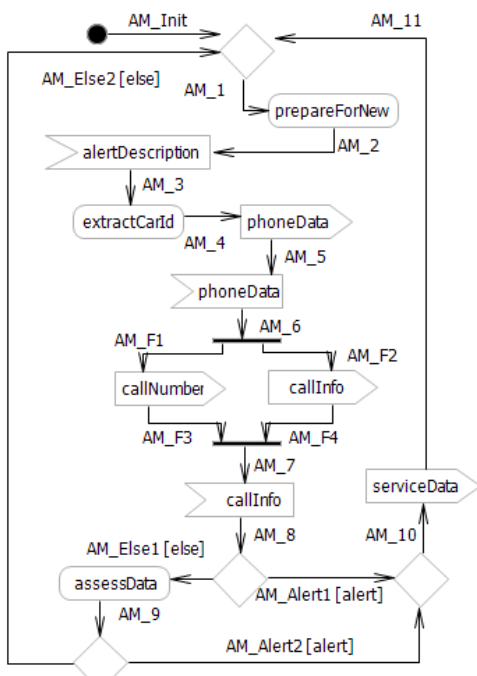

Fig. 3. Activity Diagram of the AccidentManager component

\section{Model Refactoring}

With the current architecture scalability issues may arise. Assuming that $70 \%$ of the incoming alerts are not real emergencies, the analysis of 'false alerts' consumes considerable resources. The AccidentManager may thus turn out to be a bottleneck in the system.

To address this scalability problem we extract the initial handling of alerts from the AccidentManager into an AlertListener component. The solution is depicted in Figure 4. The AlertListener receives alerts from cars, forwards them to the AccidentManager for processing while querying the database for the phone number and invoking the telephone service, which sends the results of its calls to the AccidentManager. 


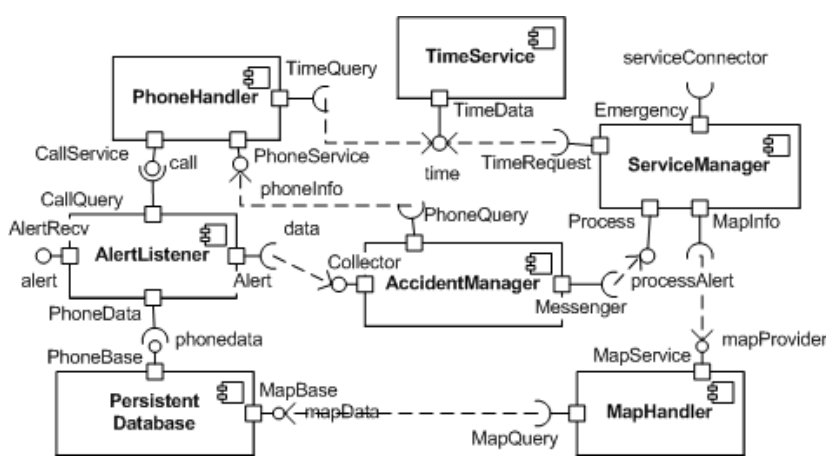

(a) Component Diagram

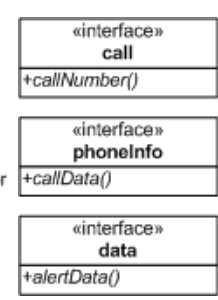

(b) Interfaces

Fig. 4. Architectural model of the refactored Accident Server

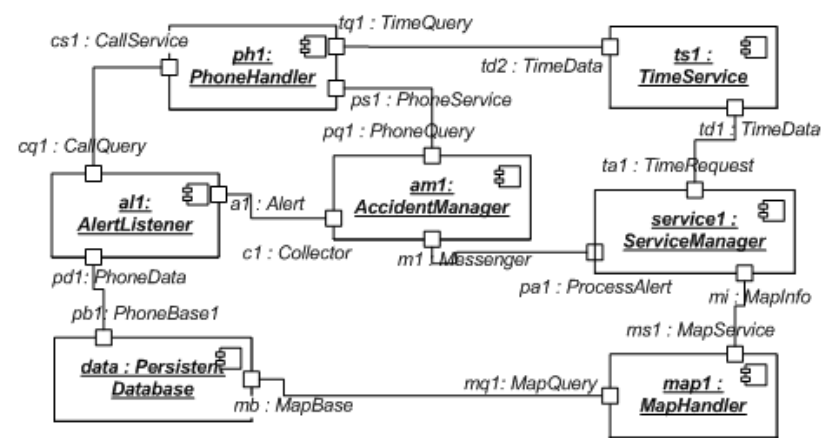

Fig. 5. Configuration after the refactoring

The behaviour of the new AlertListener component is given in Figure 6(a) while the updated behaviour of the AccidentManager is shown in Figure 6(b)

However, rather than comparing the semantics of the entire system model before and after the change, we focus on the affected parts and their immediate context. More precisely, we are proposing to extract a model transformation rule which, (1) when applied to the source model produces the target model of the refactoring and (2) is semantics preserving in the sense that its left-hand side is in the desired semantic relation with its right-hand side. We will demonstrate in Sect. 6] that this is indeed sufficient to guarantee the corresponding relation between source and target model. In the example present, such a rule is shown in Fig. 7 for the structural part only. The behaviour transformation is given by the new and updated activity diagrams associated with the components in the rule. The rule is applied by selecting in the source model an occurrence isomorphic to the left-hand side of the rule at both type and instance level. Thus, component $C$ is matched by AccidentManager from Fig. 1(a), interface $N$ corresponds to phone, $M$ to processAlert, and $J$ to phoneData. At instance level a similar correspondence is established. 


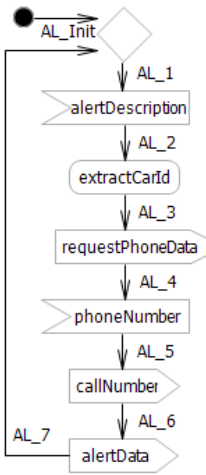

(a) AlertListener

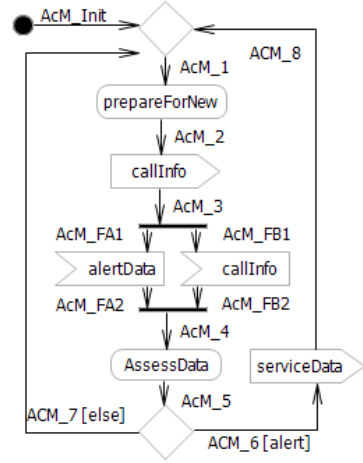

(b) AccidentManager

Fig. 6. Owned behaviour after the refactoring

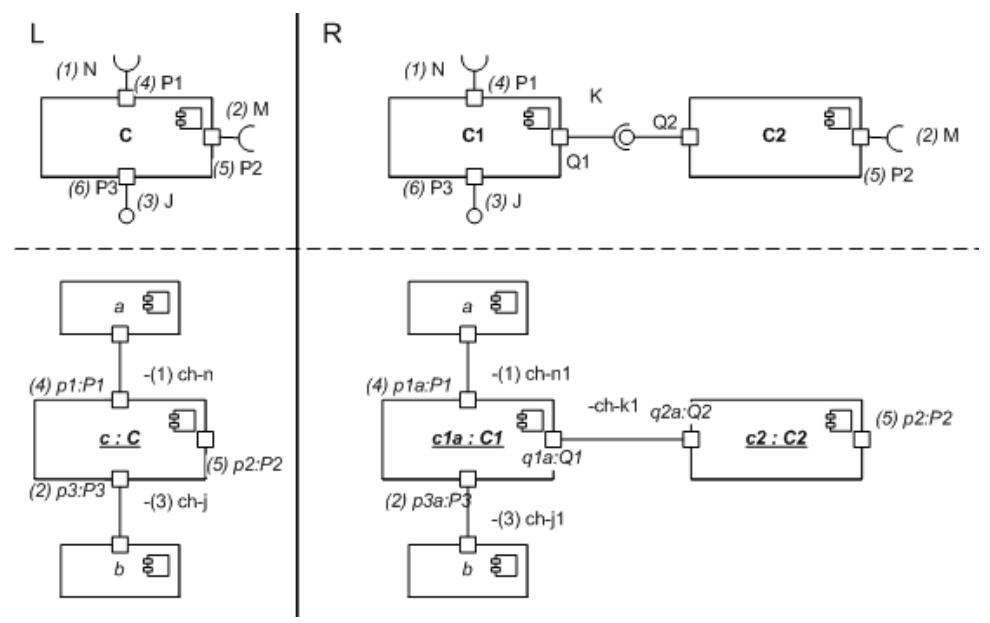

Fig. 7. Refactoring rule

A rule is extracted as follows: $G$ denotes the original model while $H$ denotes the refactored one. The smallest consistent submodel of $G$ containing $G \backslash H$ would form the left-hand side $L$ of the rule, while the smallest submodel of $H$ containing $H \backslash G$ would form the right hand side $R$. In the algebraic approach to graph transformation, which provides the formal background of this work, this is known as Initial Pushout Construction [EEPT06.

Recently a similar construction has been used as part of the model transformation by example approach, where a transformation specification is derived inductively from a set of sample transformation rules Var06. Notice that while the rule thus obtained is known to achieve the desired transformational effect, it is not in general guaranteed that the semantic relation between $L$ and $R$ can 
indeed be verified, even if it holds between $G$ and $H$. The reason is that additional context information present in $G$ and $H$ may be required to ensure semantic compatibility. It is the responsibility of the modeller to include this additional context into the rule. However, as in the example presented, a minimal rule might not be enough because some additional context may have to be taken into account in order to guarantee the preservation of the semantics. In the example this has led to the introduction into the rule of generic component instances $a$ and $b$ (the PhoneHandler and Database in the concrete model).

The example illustrates the potential complexity of the problem at hand, with changes in all three diagrams types to be coordinated in order to lead to an equivalent behaviour. In the following section we will see how the combined effect of these three models is reflected in the semantic mapping to CSP.

\section{$5 \quad$ Semantic Mapping}

In order to verify the semantic relation between source and target models, UML models are mapped to CSP processes. After introducing the relevant concepts, the mapping rules are sketched. Formally, the UML models are instances of metamodels represented by attributed typed graphs. Also, the mapping consists of rules of a triple graph grammar Sch94 presented here using the concrete syntax of UML and CSP, rather than the abstract graph-based presentation. This relation has been explained at length in [BH07], where a similar technique is used to describe the mapping of activity diagrams to CSP.

Communicating Sequential Processes. Communicating Sequential Processes Hoa85 is a process algebra providing for concurrent systems and supported by tools [FSEL05]. A process is the behaviour pattern of a component with an alphabet of events. Processes are defined using recursive equations based on the following syntax.

$$
P::=\text { event } \rightarrow P|P \sqcap Q| P \square Q|P \| Q| P \backslash a|S K I P| S T O P
$$

The prefix $a \rightarrow P$ performs action $a$ and then behaves like $P$. The processes $P \sqcap Q$ and $P \square Q$ represent internal and external choice between processes $P$ and $Q$, respectively. The process $P \| Q$ behaves as $P$ and $Q$ engaged in a lock-step synchronisation. Hiding $P \backslash a$ behaves like $P$ except that all occurrences of event $a$ are hidden. $S K I P$ represents successful termination, $S T O P$ is a deadlock. Due to the distinction of type and instance level, in our application it is important to define groups of processes with similar behaviour. To this end, we use labelling: Each process within a group is labelled by a different name, which is also used to distinguish its events. A labelled event is a pair $l . x$ where $l$ is a label, and $x$ is the event. A process $P$ labelled by $l$ is denoted by $l: P$ Hoa85].

The semantics of CSP is defined in terms of traces, failures, and divergences Hoa85. A trace of a process behaviour is a finite sequence of events in which the process has engaged up to some moment in time. The complete set of all possible traces of process $P$ is denoted by $\operatorname{traces}(P)$. For the three semantics 
domains, corresponding equivalence and refinement relations can be deducted. Two processes are trace equivalent, i.e. $P \equiv_{T} Q$ if the traces of $P$ and $Q$ are the same, i.e. $\operatorname{traces}(P)=\operatorname{traces}(Q)$. Trace refinement means that $P \sqsubseteq_{T} Q$ if $\operatorname{traces}(Q) \subseteq \operatorname{traces}(P)$. Hence, every trace of $Q$ is also a trace of $P$. Analogously the equivalence and refinement relations based on failures and divergences can be defined. These relations shall be used to express behaviour preservation of refactoring rules and compatibility of system components.

Despite the existence of more expressive mathematical models, the compositional property and tool support are most important to our aim. FDR2 [FSEL05] enables the automatic verification of the above mentioned equivalence and refinement relations.

Type-Level Mapping. In Fig. 8 the mapping of a component and its ports are shown. The component is mapped to a process definition, where its owned behaviour (obtained from the activity diagram) and the derived processes of the contained ports are put in parallel.

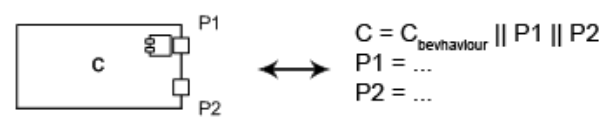

Fig. 8. Mapping of a Component and its Ports

The ports are then mapped to simple processes, allowing all send and receive events for messages declared in the interfaces that the port implements. In case of a provided interface, the port receives the messages, which is denoted by a recv $v_{\text {methodname }}$ event. For required interfaces, the port sends the message, leading to a send methodname $_{\text {event. }}$

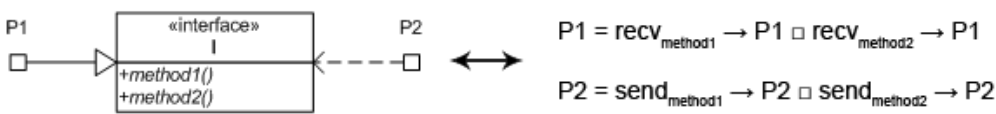

Fig. 9. Mapping of an Interface

Instance-Level Mapping. In the mapping of component instances it is important to deal with multiple occurrences. Thus, components and port instances are labelled by their instance name from the diagram as shown in Fig. 10.

As channels describe communication, they contain events corresponding to messages sent and received. As shown in Figure 11, send and recv events are labelled by the corresponding port instances and put in a prefix relationship to ensure the correct order.

As behind the concrete syntax the rules are graph transformation rules, the mapping of events between LHS and RHS graphs are notated by a unique map id. 


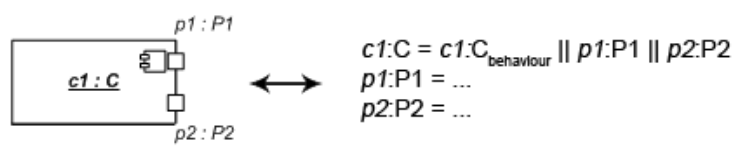

Fig. 10. Mapping of a Component Instance
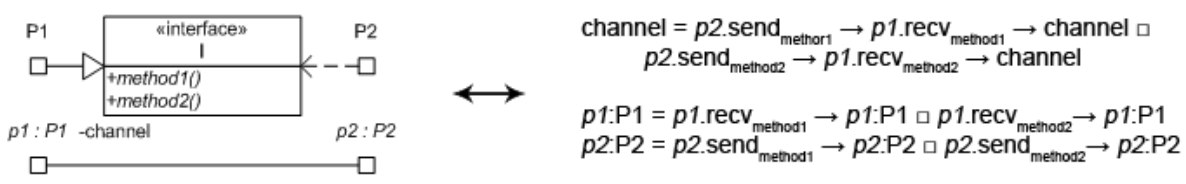

Fig. 11. Mapping of a Channel with Ports

Mapped objects bear the same map id. To make the mapped event names identical on both sides, these events are labelled on instance level by their map id, that overrides the structure based labelling presented above.

Application to the Rule. To verify the compatibility of the rule with a semantic relation, say trace refinement, we map the instance levels of both left- and righthand side to their semantic representation and verify the relation between them. For the left-hand side, for example, this yields

$$
\operatorname{sem}(L)=\mathrm{a} \| \text { ch-n }\|\mathrm{c}: \mathrm{C}\| \mathrm{ch}-\mathrm{j} \| \mathrm{b} \backslash\left\{\text { unmapped }_{1}, \text { unmapped }_{2}, \ldots\right\}
$$

by placing all component instances and connectors in parallel and hiding the unmapped events.

That means connectors and component behaviours are running in parallel. Since the parallel operator in CSP means lock-step synchronisation, whenever a send event happens at the component, the channel attached changes state to and waits for the corresponding recv event at the other end.

On the right hand side we hide all internal communication between instances of $C 1$ and $C 2$. For example, referring to our activity diagram in Fig. 6(a), the alertData and callStarted events are hidden because they serve the combination between the two parts of the newly split component $C$. To check if $\operatorname{sem}(L) \sqsubseteq$ $\operatorname{sem}(R)$ we would take into account the CSP mappings of all activity diagrams of components involved in the transformation.

Implementation. The transformation was implemented using the Tiger EMF Transformer Tig07 tool. It consists of 43 rules organised in 4 major groups (type-level, owned behaviour, instance-level, renaming). The production rules are defined by rule graphs, namely a left-hand side (LHS), a right-hand side (RHS) and possible negative application conditions (NACs). These rule graphs are object-structures that contain objects typed over EMF metamodels of UML diagrams as well as CSP expressions. These object-structures are also essentially attributed typed graphs. 
The generated CSP code for the refactoring rule was 62 lines for the LHS, and 76 lines for the RHS. The trace refinement was verified with FDR2 through 24,168 states and 121,586 transitions.

\section{Correctness of Rule-Level Verification}

In this section we demonstrate that the method of verifying a transformation by verifying an extracted rule is indeed correct. The crucial condition is the compositionality of the semantic mapping, which guarantees that the semantic relation $\mathcal{R}$ (think refinement or equivalence) is preserved under embedding of models. We will first formulate the principle and prove that, assuming this property, our verification method is sound. Then we establish a general criterion for compositionality and justify why this applies to our semantic mapping.

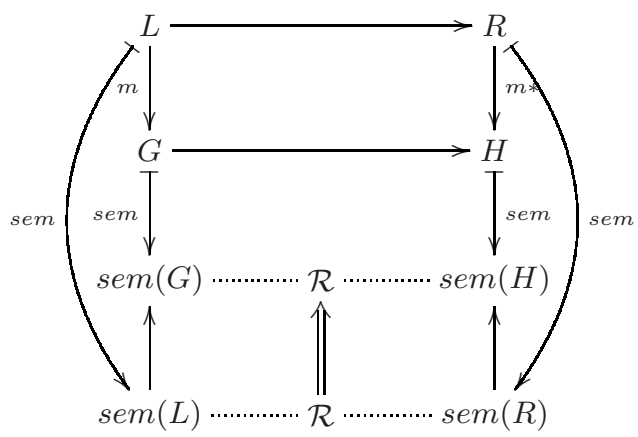

Fig. 12. CSP correspondence for behaviour verification

Correctness. The overall structure is illustrated in Fig. 12, The original model (component, composite structure and activity diagrams) is given by graph $G$. The refactoring results in graph $H$ by the application of rule $p: L \rightarrow R$ at match $m$. Applying the semantic mapping sem (itself described by a graph transformation from models to CSP) to the rule's left- and right-hand side, we obtain the CSP processes $\operatorname{sem}(L)$ and $\operatorname{sem}(R)$. Whenever the relation $\operatorname{sem}(L) \mathcal{R} \operatorname{sem}(R)$ (say $\mathcal{R}=\sqsubseteq$ is trace refinement, so all traces of the left processes are also traces of the right), we would like to be sure that also $\operatorname{sem}(G) \mathcal{R} \operatorname{sem}(H)$ (traces of $\operatorname{sem}(G)$ are preserved in $\operatorname{sem}(H))$.

The main assumption is the compositionality of the semantic mapping sem. Intuitively, the mapping must be closed under context, i.e., the semantics of a model $L$ is embedded within the semantics of an extension $G$ of $L$. Embedding of CSP processes is expressed by the notion of context, i.e., a CSP expression with a single occurrence of a distinguished free variable.

Definition 1 (compositionality). A mapping sem from graphs to CSP processes is compositional if for each injective graph morphisms $m: L \rightarrow G$ there 
exists a context $E$ such that $\operatorname{sem}(G) \equiv E(\operatorname{sem}(L))$. Moreover, this context is uniquely determined by the part of $G$ not in the image of $L$, i.e., given a pushout diagram as below with injective morphisms only, and a context $F$ with $\operatorname{sem}(D) \equiv F(\operatorname{sem}(K))$, then $E$ and $F$ are equivalent.

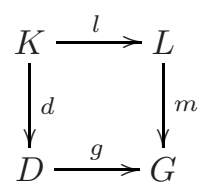

Definition 1 applies particularly where $L$ is the left hand side of a rule and $G$ is the given graph of a transformation. In this case, the CSP expression generated from $L$ contains the one derived from $G$ up to traces, failures or divergences equivalence, while the context $E$ is uniquely determined by $G \backslash m(L)$. The proof also relies on the fact that semantic relations $\mathcal{R}$ in CSP are closed under context.

Theorem 1. Assume a compositional mapping sem from graphs to CSP processes. Then, for all transformations $G \stackrel{p, m}{\Longrightarrow} H$ via rule $p: L \rightarrow R$ with injective match $m$, it holds that $\operatorname{sem}(L) \mathcal{R} \operatorname{sem}(R)$ implies $\operatorname{sem}(G) \mathcal{R} \operatorname{sem}(H)$, where $\mathcal{R}$ may be any of $\{\equiv, \sqsubseteq, \sqsupseteq\}$ on traces, failures, or divergences.

Proof. By assumption the match $m$, and therefore the comatch $m^{*}: R \rightarrow H$ are injective. Since the mapping sem is compositional, according to Definition 1 there are contexts $E$ and $F$ such that $\operatorname{sem}(G) \equiv E(\operatorname{sem}(L))$ and $\operatorname{sem}(H) \equiv$ $F(\operatorname{sem}(R))$. Now, $E(\operatorname{sem}(L)) \mathcal{R} E(\operatorname{sem}(R))$ since $\operatorname{sem}(L) \mathcal{R} \operatorname{sem}(R)$ and $\mathcal{R}$ is closed under context. Finally, $E(\operatorname{sem}(R)) \equiv F(\operatorname{sem}(R))$ by the uniqueness of the contexts.

Thus, it remains to demonstrate that compositionality holds for the semantic mapping at hand. The proof is based on the embedding and extension theorem for double-pushout graph transformation [EHK01] and only sketched below. Full details are given in Bis08.

Compositionality. In this section we present a proof sketch for the fact that our semantic mapping is compositional. The result is interesting by itself because it holds for a large class of mappings described by triple graph grammars Sch94. The idea is that triple graph grammars describe model transformations by creating the target from the source model and linking both by a relation model. Hence it is not necessary to remove the source model, and rules can be designed in such a way that also on the relation and target model rules are non-deleting.

For simple rules (without negative application conditions), compositionality then follows directly from the fact that the transformations realising the semantic mapping can be embedded into larger contexts.

Theorem 2. Given a mapping sem from graphs to CSP described by graph transformation rules in the DPO approach. If all rules of sem are non-deleting and do not contain negative application conditions, then the mapping is compositional. 
Proof. (sketch) The main argument is based on the Embedding Theorem EEPT06 applied to a transformation sequence $t=G_{0} \stackrel{*}{\Rightarrow} G_{n}$ implementing the mapping. We assume a graph $H_{0}$ containing $G_{0}$ with inclusion morphism $m_{0}$. For a transformation $t$ we create a boundary graph $B$ and a context graph $C$. The boundary graph is the smallest subgraph of $G_{0}$ which contains the identification points and dangling points of $m_{0}$. Since (2) is a pushout, the context graph can be determined. If none of the productions of $t$ deletes any item of $B$, then $m_{0}$ is consistent with $t$ and there is an extension diagram over $t$ and $m_{0}$. This means that $H_{n}$ is the pushout complement of $t$ and $m_{0}$, and can thus be determined without applying the transformation $t$ on $H_{0}$. Hence, the compositionality condition holds for $c$.

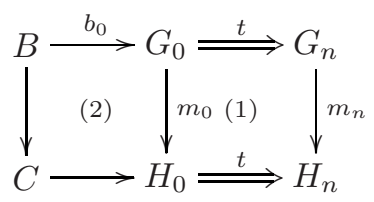

Adding context and boundary graph to our picture, we get the extension diagram above.

The initial graph $G_{0}$ is either the left- or right-hand side of our refactoring rule. Graph $G_{n}$ thus corresponds to either $\operatorname{sem}(L)$ or $\operatorname{sem}(R)$, i.e., our graph transformation $t$ implements the mapping sem. The inclusion into $H_{0}$ is given by the match $m_{0}: L \rightarrow G$ for the refactoring rule.

Negative application conditions (NAC) restrict the applicability of rules by forbidden patterns, i.e., structures which must not occur in the given graph in order for the transformation to be permitted. The definition of our mapping, as many realistic transformation systems, make heavy use of this feature. In order to extend Theorem 2, we require the notions of created points and creational type. Intuitively, created points are those nodes and edges in a graph production rule that are only present in the right-hand side, and are thus created during its application. The creational type is the set of all nodes and edges in the type graph (metamodel) that are never deleted during a transformation. Formal definitions are given in Bis08, as well as the proof of the theorem.

Theorem 3. A mapping sem from graphs to CSP is compositional if

1. All rules of sem are non-deleting;

2. All NACs defined for rules in sem only contain elements of created types;

3. Source models do not contain any elements of created types.

These conditions are naturally satisfied in the case of triple graph grammars Sch94: Created types are elements of the target and relational metamodels, hence they do not occur in source models. The only real restriction is that no negative application conditions are allowed on the source model. Our mapping from UML architectural models to CSP satisfies these restrictions and is thus compositional. 


\section{Conclusion and Future Work}

The results presented in this paper are spanning two levels of abstraction. At the level of architectural refactoring, we have developed a method for verifying transformations of UML architectural models based on a semantic mapping into CSP processes. More generally, we have shown that the correctness of such an approach depends on the compositionality of the semantic mapping, and that this property can be guaranteed by a structural condition on the form of the mapping rules which is easily satisfied, for example, by triple graph grammars.

Future work will continue to address both levels. At the concrete level we hope to be able to come up with a catalogue of verified refactoring rules, rather than relying on the extraction of rules from individual transformations as in this paper. It remains to be seen if a general catalogue comparable to OO refactorings is possible. In general, the approach of rule extraction needs to be supported by heuristics about which elements of a model, apart from those that are changed, should be included into the rule in order to verify its semantic compatibility.

\section{References}

ADG98. Allen, R., Douence, R., Garlan, D.: Specifying and Analyzing Dynamic Software Architectures. In: Astesiano, E. (ed.) FASE 1998. LNCS, vol. 1382, Springer, Heidelberg (1998)

BH07. Bisztray, D., Heckel, R.: Rule-level verification of business process transformations using csp. In: Proc. of 6th International Workshop on Graph Transformations and Visual Modeling Techniques (GTVMT 2007) (2007)

Bis08. Bisztray, D.: Verification of architectural refactoring rules. Technical report, Department of Computer Science, University of Leicester (2008), http://www.cs.le.ac.uk/people/dab24/refactoring-techrep.pdf

EEPT06. Ehrig, H., Ehrig, K., Prange, U., Taentzer, G.: Fundamentals of Algebraic Graph Transformation. EATCS Monographs in Theoretical Computer Science. Springer, Heidelberg (2006)

EHK01. Engels, G., Heckel, R., Küster, J.M.: Rule-based specification of behavioral consistency based on the UML meta model. In: Gogolla, M., Kobryn, C. (eds.) UML 2001. LNCS, vol. 2185, Springer, Heidelberg (2001)

EKGH01. Engels, G., Küster, J.M., Groenewegen, L., Heckel, R.: A methodology for specifying and analyzing consistency of object-oriented behavioral models. In: Gruhn, V. (ed.) Proc. European Software Engineering Conference (ESEC/FSE 2001), Vienna, Austria. LNCS, vol. 1301, pp. 327-343. Springer, Heidelberg (2001)

$\mathrm{FBB}^{+}$99. Fowler, M., Beck, K., Brant, J., Opdyke, W., Roberts, D.: Refactoring: Improving the Design of Existing Code, 1st edn. Addison-Wesley Professional, Reading (1999)

FSEL05. Formal Systems Europe Ltd. FDR2 User Manual (2005), http://www.fsel.com/documentation/fdr2/html/index.html

HIM98. Hirsch, D., Inverardi, P., Montanari, U.: Graph grammars and constraint solving for software architecture styles. In: ISAW 1998: Proceedings of the third international workshop on Software architecture, pp. 69-72. ACM Press, New York (1998) 
Hoa85. Hoare, C.A.R.: Communicating Sequential Processes. Prentice Hall International Series in Computer Science. Prentice Hall, Englewood Cliffs (1985)

Lae25. Laertius, D.: Lives of Eminent Philosophers, vol. 2. Loeb Classical Library (January 1925)

LM04. Leino, K.R.M., Müller, P.: Object invariants in dynamic contexts. In: Odersky, M. (ed.) ECOOP 2004. LNCS, vol. 3086, pp. 491-516. Springer, Heidelberg (2004)

MDJ02. Mens, T., Demeyer, S., Janssens, D.: Formalising behaviour preserving program transformations. In: Corradini, A., Ehrig, H., Kreowski, H.-J., Rozenberg, G. (eds.) ICGT 2002. LNCS, vol. 2505, pp. 286-301. Springer, Heidelberg (2002)

MGB06. Massoni, T., Gheyi, R., Borba, P.: An approach to invariant-based program refactoring. In: Software Evolution through Transformations 2006, Electronic Communications of the EASST (2006)

MK96. Magee, J., Kramer, J.: Dynamic structure in software architectures. In: SIGSOFT 1996: Proceedings of the 4th ACM SIGSOFT symposium on Foundations of software engineering, pp. 3-14. ACM Press, New York (1996)

OMG06. OMG. Unified Modeling Language, version 2.1.1 (2006), http://www.omg.org/technology/documents/formal/uml.htm

Sch94. Schür, A.: Specification of graph translators with triple graph grammars. In: Mayr, E.W., Schmidt, G., Tinhofer, G. (eds.) WG 1994. LNCS, vol. 903, pp. 151-163. Springer, Heidelberg (1995)

Sel98. Selic, B.: Using uml for modeling complex real-time systems. In: Müller, F., Bestavros, A. (eds.) LCTES 1998. LNCS, vol. 1474, pp. 250-260. Springer, Heidelberg (1998)

SPTJ01. Sunyé, G., Pollet, D., Le Traon, Y., Jézéquel, J.-M.: Refactoring uml models (2001)

TGM00. Taentzer, G., Goedicke, M., Meyer, T.: Dynamic change management by distributed graph transformation: Towards configurable distributed systems. In: Ehrig, H., Engels, G., Kreowski, H.-J., Rozenberg, G. (eds.) TAGT 1998. LNCS, vol. 1764, pp. 179-193. Springer, Heidelberg (2000)

Tig07. Tiger Developer Team. Tiger EMF Transformer (2007), http://www.tfs.cs.tu-berlin.de/emftrans

Var06. Varró, D.: Model transformation by example. In: Nierstrasz, O., Whittle, J., Harel, D., Reggio, G. (eds.) MoDELS 2006. LNCS, vol. 4199, pp. 410-424. Springer, Heidelberg (2006)

$\mathrm{WCG}^{+}$06. Wirsing, M., Clark, A., Gilmore, S., Hölzl, M., Knapp, A., Koch, N., Schroeder, A.: Semantic-Based Development of Service-Oriented Systems. In: Najm, E., Pradat-Peyre, J.-F., Donzeau-Gouge, V.V. (eds.) FORTE 2006. LNCS, vol. 4229, pp. 24-45. Springer, Heidelberg (2006)

WF02. Wermelinger, M., Fiadeiro, J.L.: A graph transformation approach to software architecture reconfiguration. Sci. Comput. Program. 44(2), 133-155 (2002) 